\title{
Automatic Autonomous Vision-based Power Line Inspection: A Review of Current Status and the Potential Role of Deep Learning
}

\author{
Van Nhan Nguyen ${ }^{\mathrm{a}, \mathrm{b}, *}$, Robert Jenssen $^{\mathrm{a}}$, Davide Roverso ${ }^{\mathrm{b}}$ \\ ${ }^{a}$ UiT Machine Learning Group, Faculty of Science and Technology, Department of Physics and Technology, University of Troms $\phi$, 9019 Troms $\phi$, Norway \\ ${ }^{b}$ Analytics Department, eSmart Systems, 1783 Halden, Norway
}

\begin{abstract}
To maintain the reliability, availability, and sustainability of electricity supply, electricity companies regularly perform visual inspections on their transmission and distribution networks. These inspections have been typically carried out using foot patrol and/or helicopter-assisted methods to plan for necessary repair or replacement works before any major damage, which may cause power outage. This solution is quite slow, expensive, and potentially dangerous. In recent years, numerous researches have been conducted to automate the visual inspections by using automated helicopters, flying robots, and/or climbing robots. However, due to the high accuracy requirements of the task and its unique challenges, automatic vision-based inspection has not been widely adopted. In this paper, with the aim of providing a good starting point for researchers who are interested in developing a fully automatic autonomous vision-based power line inspection system, we conduct an extensive literature review. First, we examine existing power line inspection methods with special attention paid to highlight their advantages and disadvantages. Next, we summarize well-suited tasks and review potential data sources for automatic vision-based inspection. Then, we survey existing automatic vision-based power line inspection systems. Based on that, we propose a new automatic autonomous vision-based power line inspection concept that uses Unmanned Aerial Vehicle (UAV) inspection as the main inspection method, optical images as the primary data source, and deep learning as the backbone of data analysis and inspection. Then, we present an overview of possibilities and challenges of deep vision (deep learning for computer vision) approaches for both UAV navigation and UAV inspection and discuss possible solutions to the challenges. Finally, we conclude the paper with an outlook for the future of this field and propose potential next steps for implementing the concept.
\end{abstract}

Keywords: Power line inspection, vision-based inspection, deep learning, UAVs

\section{Introduction}

The increasing dependence of modern-day societies on electricity poses corresponding challenges on the monitoring, inspection, and maintenance of the electric power grids to ensure uninterrupted flow of electricity. Due to the lack of incentives to invest in aged national grid infrastructures, for example, in Europe and the US, power cuts are becoming more and more frequent [1]. While short-term power failures typically last only a few hours, long-term blackouts can last days or even weeks. Power outages, both short and long-term, can have catastrophic effects on unprepared businesses. For instance, blackouts can completely shut down production at companies and critical infrastructures such as telecommunication networks, financial services, water supplies, and hospitals [2]. Nowadays, most of the power grids are interconnected. Hence, a blackout in one region can trigger a domino effect that could result in supraregional blackouts [3]. According to [1], a 30-minute power cut in the US results in an average loss of US\$15,709 for medium

\footnotetext{
${ }^{*}$ Corresponding author.

Email addresses: nhan.v.nguyen@uit.no (Van Nhan Nguyen), robert.jenssen@uit.no (Robert Jenssen),

Davide.Roverso@esmartsystems . com (Davide Roverso)
}

and large industrial clients, and nearly US\$94,000 for an eighthour interruption. Thus, power grids are required to be monitored, inspected, and maintained regularly to prevent faults which may cause power failures (Fig. 1 and 2).
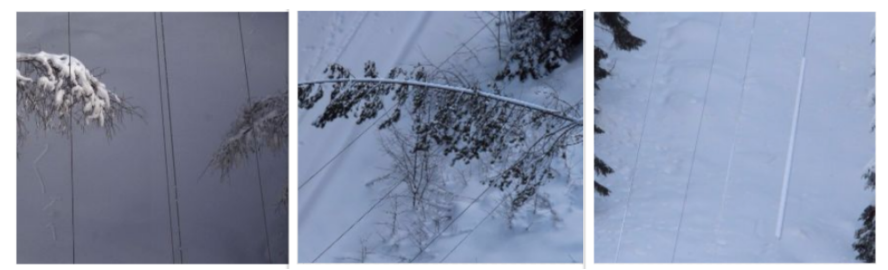

Figure 1: Common faults on power lines (from left to right): trees growing too close to power lines, trees lying across power lines, icing on power lines. In warm countries, icing on power lines may not be a relevant fault; however, in cold countries, such as Norway, it is a very serious problem since thick icing accumulated on power lines can cause a great deal of damage to the lines.

The traditional methodologies for inspecting power networks typically include field surveys and airborne surveys, which have been unchanged for decades [4]. On a regular basis or in emergency situations, such as storms, hurricanes, and earthquakes, teams of inspectors are sent out, travelling either on foot or by helicopters, to visually inspect the power lines with the help of binoculars and sometimes with infrared (IR) and corona de- 
tection cameras [5]. The main reason why visual inspection is popular is that it can cover a wide range of common faults on both power lines components (Fig. 2) and the power lines themselves (Fig. 1) in one inspection. However, this methodology is quite slow, expensive, potentially dangerous, and with detection rate limited by the visual observation skills of inspectors. Although digital cameras can be used to separate the data collection from data analysis, both processes have still been performed manually.
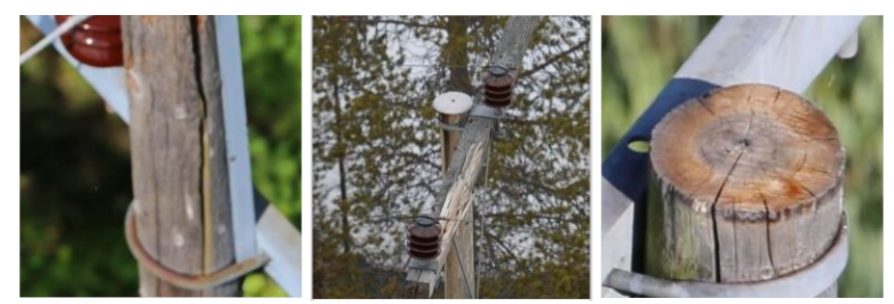

Figure 2: Common faults on power lines components (from left to right): broken poles, broken crossarms, missing toppads. The toppads are used for protecting wooden poles from rain. In Norway, missing toppads is considered a fault; however, in the US, it may not be considered as a fault since toppads are not widely applied.

Over the past few years, many studies have been conducted to automate the visual inspection by using automated helicopters, flying robots, and/or climbing robots; however, there are very few published reviews of different approaches to the problem, including vision-based approaches. In this paper, with the aim of providing a comprehensive overview of the possibilities and challenges of automatic autonomous vision-based inspection of power lines, we conduct an extensive literature review. First, we examine existing power line inspection methods including foot patrol, helicopter-assisted, automated helicopterassisted, climbing robots, and unmanned aerial vehicles (UAVs) inspection with special attention paid to highlight their advantages and disadvantages. Next, we summarize inspection tasks which are well suited for automatic autonomous vision-based inspection: mapping and inspection of power line components, vegetation encroach monitoring, icing detection and measurement, and disaster monitoring. Then, we review potential data sources for vision-based inspection including synthetic aperture radar (SAR) images, optical satellite images, optical aerial images, thermal images, ultraviolet images, airborne laser scanner (ALS) data, land-based mobile mapping data, and UAV data and point out their applicable tasks. Further, we conduct a comprehensive review of current automatic vision-based power line inspection systems.

Based on that, we propose as a new potential solution an automatic autonomous vision-based power line inspection concept that uses UAV as the main inspection method, optical images as the primary data source, and deep learning as the backbone of data analysis and inspection.

To facilitate the implementation of the concept, we first discuss the potential role of deep learning in automatic autonomous vision-based power line inspection. We then highlight the possibilities and challenges of deep vision (deep learning for computer vision) approaches for both UAV navigation and UAV inspection. Finally, we propose possible solutions to the challenges and suggest potential next steps.

The remainder of the paper is structured as follows: Section 2 summarizes existing relevant literature reviews (related work) including inspection with vision-based approaches, before we present a brief introduction to power line inspection in general together with a summary of different inspection methods, inspection tasks, and data sources for visual inspection in Section 3. Next, in Section 4, we present a comprehensive review of existing vision-based approaches for both UAV navigation and UAV inspection. Finally, in Section 5 and Section 6, we identify the remaining challenges of vision-based approaches in automatic inspection, discuss possible solutions to these challenges, propose potential next steps for implementing the automatic autonomous vision-based power line inspection concept, and conclude the paper with a summary and an outlook for the future of the field.

\section{Related Work}

Although vision-based inspection is one of the most promising approaches for reducing or completely eliminating people, in both data collection and analysis, there are very few published reviews of vision-based approaches for power line inspections.

A review of recent techniques for the vegetation encroachment monitoring was presented in [6]. According to the authors, current methods for vegetation monitoring including visual field inspection, aerial video surveillance, aerial multispectral imaging, and LiDAR scanning are not reliable due to their high cost, inaccuracy, and high time complexity. To increase the reliability of vegetation monitoring, satellite stereo and wireless multimedia sensor networks (WMSNs) were proposed to be the two future technologies. Based on that, the authors discussed the concept of utilizing multispectral satellite stereo images and WMSNs in detecting dangerous vegetation.

Mirallès François et al. conducted a survey of computer vision applications in power line inspection in [7]. The surveyed applications include detection of power lines, inspection of power lines, detection and inspection of insulators, power line corridor maintenance, and pylon detection. According to the authors, computer vision appears to be one of the most important technologies for automatic vision-based power line inspection since both robots and UAVs need it not only for guidance, navigation, and control but also for inspection. Although this technology has been facilitating some applications, for example vegetation monitoring, it has not been widely used for other important applications such as inspection of defects on the cables or insulators.

Recent advantages in remote sensing sensors and data analysis approaches have opened new possibilities in automatic power line inspection. To facilitate the use of remote sensing techniques in power line inspection, a very comprehensive survey of the possibilities of modern remote sensing sensors for automatic power inspection was conducted in [4]. The authors summarized main applications, advantages, and challenges of different remote sensing data sources for power line inspection 
including SAR images, optical satellite and aerial images, thermal images, airborne laser scanner data, mobile mapping data, and UAV data. The authors pointed out that while mapping and analysis of network components is the main focus of most of the previous studies, vegetation monitoring has been receiving less attention. Since each remote sensing approach has its own specific advantages and disadvantages, the authors suggested that more attention should be given to the use of use multiple data sources fusion in the future research to benefit from various approaches in an optimal way.

\section{Vision-based Power Line Inspection}

In this section, we first review existing power line inspection methods with special attention paid to highlight their advantages and disadvantages. Next, we summarize power line inspection tasks that are well suited for automatic autonomous vision-based inspection. Then, we survey potential data sources for vision-based power line inspection and point out their applicable inspection tasks. Finally, we give a brief introduction to automatic vision-based power line inspection systems and their required components.

\subsection{Power Line Inspection Methods \\ 3.1.1. Foot Patrol Inspection}

Foot patrol inspection is typically conducted by a team of usually two inspectors traveling on foot to visually inspect the power lines with the help of binoculars and sometimes with Infrared cameras and corona detection cameras [5]. The main disadvantage of this inspection method is that it is very slow, tedious, and labor-insensitive. Another major drawback of this method is that it may not be possible in harsh terrains, in extreme weather conditions, and after extreme events, such as hurricanes, wind storms, and snow storms. However, despite its disadvantages, foot patrol inspection has still been widely applied because of its high detection rate [4].

\subsubsection{Helicopter-assisted Inspection}

In helicopter-assisted inspection, a team of usually three (or two) people including a pilot, an inspector, and/or a camera operator is sent out traveling by helicopter to perform online inspection and acquire data for offline inspection. The inspector is responsible for identifying obvious faults, for example trees growing too close to power lines, trees lying across or against power lines, and collapsed poles. The camera operator films the conductors, pylons, power components (e.g., insulators, transformers, crossarms, toppads), and objects around the pylons and under the lines. After the flight, an offline inspection is typically conducted by a team of inspectors manually browsing through the collected videos and images to identify more complicated and smaller faults, such as broken insulators, missing toppads, and missing splints. Although this inspection method allows access to hard-to-reach locations and increases inspection speed, it comes with many disadvantages, such as high cost, low accuracy due to high speeds, the dependence on human visual observation skills, and always there is a risk of contacting live lines and loss of lifes [8].

\subsubsection{Automated Helicopter-assisted Inspection}

Automated helicopter-assisted inspection is quite similar to the helicopter-assisted inspection method. The main difference is that the former method utilizes vision-based approaches in both data collection and and data analysis. Vision-based approaches, for example power mast detection, can be applied for guiding cameras to automatically film the conductors, pylons, power components, and objects around the pylons and under the power lines. The acquired videos and images can be later analyzed automatically by vision-based data analysis approaches. Although automated helicopter-assisted inspection can reduce the dependence on human visual observation skills and increases inspection speed, it has not been widely applied due to high inspection cost, safety issues, and challenges of applying vision-based approaches in power line inspection.

\subsubsection{Climbing Robots Inspection}

In climbing robots inspection, the inspection is conducted by a robot traveling on power lines. The robot is typically equipped with many sensors and cameras (e.g., visual cameras, thermal cameras) for navigating along power lines, crossing obstacles on the lines, and inspecting the lines and power components. The main advantage of this method is the inspection accuracy due to its proximity to the power lines. However, according to [9], the weight of the robots could damage the lines, and the robots may not be able to pass across various obstacles on the lines. Another major disadvantage of this inspection method is that it is relatively slow compared to other inspection methods such automated helicopter-assisted inspection or UAVs inspection.

\subsubsection{UAVs Inspection}

In UAVs inspection, the inspection is conducted by UAVs equipped with multiple sensors and cameras for navigating along power lines, performing online inspection to detect obvious faults, and collecting data for later offline inspections. UAVs inspection offers great possibilities for addressing most of the existing issues of other inspection methods such as high inspection cost, low speed, and safety. The operation costs of UAVs inspection is relative low compared to foot patrol and (automated) helicopter-assisted inspections. In addition, UAVs can fly relatively close to power lines to take detailed images of conductors, pylons, and power components, which can significantly improve inspection accuracy.

However, fully automatic autonomous power line inspection systems using UAVs still come with great challenges. For example, In UAV navigation, detection and tracking of power lines are crucial tasks; however, existing vision-based detection and tracking methods are still not accurate enough for detecting and tracking the power lines since they are typically too small leading to a lack of rich features for their representation.

\subsection{Power Line Inspection Tasks}

\subsubsection{Mapping and Inspection of Power Line Components}

Mapping and inspection of power line components, such as conductors, pylons, and power components, are among the 
most studied topics in the field of power line inspection. These tasks are typically conducted either online by, for example, a foot patrol team with the help of binoculars to manually observe and detect faults or offline by, for instance, analyzing images taken from UAVs and/or helicopters. Some common faults on power line components which can be identified by the two approaches are broken poles, broken crossarms, and missing toppads (Fig. 2). In addition to the above mentioned approaches, other data sources such as thermal images, airborne laser scanner, and land-based mobile mapping data have also been used as alternatives for mapping and inspection of power line components [4].

\subsubsection{Vegetation Encroachment Monitoring}

According to [6], one of the most frequent causes of flashovers in both transmission and distribution networks is encroachment to the power lines. In addition, maintaining safety distances between vegetation and the power lines along the corridor is a legal requirement in some countries. Thus, it is required that vegetation near power line corridors are cleared regularly to prevent power outages and damage to the power lines [10]. The main tasks of vegetation encroachment monitoring include detecting and classifying near zone vegetation, and estimating their height and their relative distance to the power lines [7].

\subsubsection{Icing Detection and Measurement}

In cold weather conditions, such as during snowstorm, hail, and freezing rain, thick icing accumulated on power lines can cause serious damage to the lines which may lead to power supply interruption [11]. One of the most important tasks in icing detection and measurement is estimating the icing thickness parameter, which is a crucial data source for energy companies to make decisions for icing accident prevention. The parameter is usually calculated by analyzing images collected from fixed monitoring terminals, which are typically mounted on power poles, for small areas. For large areas, foot patrol and helicopter-assisted inspections are usually conducted with the help of binoculars and/or telescopes to manually observe and estimate the icing thickness parameter [12].

\subsubsection{Disaster Monitoring}

Natural disasters, such as storms, hurricanes, and earthquakes, can cause a great deal of damage to power lines that could result in outages or even completely shut down whole power grids. Thus, we are in an urgent need for a fast approach for damage assessments to quickly recover the power grids after natural disasters. However, according to our review, only a few research articles have been focused on this topic.

\subsection{Data Sources for Power Line Inspection}

Inspired by the comprehensive review of different remote sensing data sources for automatic inspection conducted by Leena Matikainen et al. in [4], in this section, we briefly summarize different relevant data sources for automatic autonomous vision-based power line inspection with special attention paid to highlight their advantages and point out their potential applications.

\subsubsection{Synthetic Aperture Radar Images}

Synthetic Aperture Radars (SAR) are active imaging sensors, which are typically used for creating 2- or 3-dimensional images of objects, such as landscapes. SAR is also commonly used for change detection and 4-D mapping (space and time). SAR images are usually acquired by SARs mounted on various platforms such as small aircrafts and satellites [13]. Because SARs can provide high-resolution, day-and-night, and weatherindependent images of large areas, it has been used for various applications in power line inspection such as vegetation mapping and monitoring [4].

\subsubsection{Optical Satellite Images}

Optical satellite images are collected by passive satellite sensors in the visible and near-infrared (NIR) wavelengths. Although satellite imagery is very expensive and usually does not work well under cloudy and dark conditions, it offers many advantages for power inspection such as large-area coverage and multispectral data. According to [4], optical satellite images has been mainly used for vegetation monitoring in power line inspection.

\subsubsection{Optical Aerial Images}

Optical aerial images are usually collected by either a manned helicopter or a fixed wing aircraft mainly in the visible and near-infrared (NIR) wavelengths. With the flexibility in data acquisition and the ability to collect detailed images of conductors, pylons, power components (e.g., insulators, transformers, crossarms, toppads), and surrounding areas, optical aerial imaging has been used in many power line inspection tasks, for example vegetation monitoring, mapping of conductors and pylons, and monitoring faults in power line components [4].

\subsubsection{Thermal Images}

Thermal images are formed by thermographic cameras, which are also known as infrared cameras or thermal imaging cameras. Thermographic cameras are based on infrared radiation to extend our vision beyond the short-wavelength red into the far infrared by making visible the light naturally emitted by warm objects [14]. With that ability, thermal images have been used for fault monitoring in power line components-based measurement and analysis of relative temperature differences [4].

\subsubsection{Ultraviolet Images}

Ultraviolet (UV) images are typically formed using UVsensitive cameras (e.g., Corona 350 II system) by recording images using light from the ultraviolet spectrum only. There are two main approaches for capturing ultraviolet images: reflected ultraviolet and ultraviolet induced fluorescence photography [15]. With the ability to visualize details which are in- 
visible to the unaided human eye, images taken with ultraviolet light have been widely used for detecting corona discharges from high voltage electric power transmission lines [16].

\subsubsection{Airborne Laser Scanner Data}

Airborne Laser Scanning (ALS) is an active remote sensing technique that uses a laser beam as the sensing carrier [17]. This type of system is usually realised with the ability to obtain range images. This laser radar is also known as LADAR (LAser Detection And Ranging) and LIDAR (LIght Detection And Ranging). ALS data is a georeferenced point cloud of LIDAR measurements [18]. According to [4], ALS data is applicable for mapping of conductors, pylons, and individuals trees near power lines since ALS can produce detailed 3D data directly without the dependence on external lightning conditions.

\subsubsection{Land-based Mobile Mapping Data}

Land-based mobile mapping data is geospatial data collected by mapping sensors that are mounted on a mobile platform such as cars, all-terrain vehicles (ATV), boats, or on a backpack carried by a person. A mobile mapping system typically includes an image data collecting module (e.g., camera, laser scanner), navigation and positioning sensors (e.g., GPS), and an inertial measurement unit (IMU) [19]. The two most common types of land-based mobile mapping data used in the power line inspection literature are images and point clouds. Because of the panoramic imaging geometry and the ability to produce very detailed 3D data and images, land-based mobile mapping data has been used for mapping conductors and pylons, and for inspecting of power line components [4].

\subsubsection{Unmanned Aerial Vehicle Data}

According to [4], the two most common types of data that have been acquired by UAVs in the power line inspection literature are optical images and laser scanning data. Because of the low operation costs, the high flexibility in data acquisition, and the ability to fly relatively close to power lines to take detailed pictures, UAV data has been used for both mapping and inspection of power lines components, and for detailed mapping of vegetation.

\subsection{Automatic Vision-based Power Line Inspection}

To build an automatic autonomous vision-based power line inspection system, we need three main components: an inspection method (e.g., UAVs inspection, helicopter-assisted inspection), a primary data source (e.g., optical images, thermal images, SAR images), and a method for data analysis.

Based on the reviews presented above, we propose UAVs as a promising potential means for automatic autonomous visionbased inspection because of the following reasons: First, UAVs inspection has significantly lower operation costs compared to other inspection methods, such as helicopter-assisted inspection and foot patrol inspection. Second, UAVs have the ability to fly relatively close to power lines to take detailed pictures, which is very useful for detecting small faults, for instance broken wires and missing splints. Third, with the ability to access hard-to-reach locations with high speeds, UAVs are considered as a highly promising solution for many inspection tasks, for example for monitoring damage on power lines caused by natural disasters, or for estimating the height of near zone vegetation, for detecting dangerous surrounding objects, and for detecting and measuring icing thickness on power lines (Fig. 1). Finally, recent advances in battery and fuel cell technologies [20], sensors, and UAV components [21] have significantly improved the feasibility of UAV-based power line inspection.

We propose optical images collected by UAVs as a potential data source because (i) they are easy to collect, (ii) relatively easier to analyze than the other reviewed data sources while (iii) providing enough information for detecting a wide range of common faults on both power components (Fig. 2) and the power lines themselves (Fig. 1).

To have a closer look into different data analysis approaches for both navigation and inspection, we present a thorough literature review of current vision-based inspection systems with special attention paid to systems that are relevant to UAVs and optical images in the next section.

\section{A Review of Current Vision-based Inspection Systems}

In this section, we highlight some of the most recently published vision-based approaches which are relevant for developing fully automatic autonomous vision-based power line inspection systems using UAVs. The review is divided in two parts. The first part summarizes vision-based approaches that are suitable for UAV navigation including power line detectionbased and pole detection-based approaches. The second part is dedicated to vision-based approaches which have the potential for automating the four main UAV inspection tasks: icing detection and measurement, vegetation encroachment monitoring, mapping and inspection of power line components, and disaster monitoring.

\subsection{Vision-based Approaches for Navigation}

According to [9], there are three common approaches for UAV navigation in automatic power line inspection: GPS way points-based, pole detection-based, and power line detectionbased. While the first approach has been widely applied for decades, the other two approaches have recently been facilitated by advances in visual recognition. In this section, we focus on reviewing recently published vision-based approaches for navigation in power line inspection with special attention paid to highlight approaches which are relevant to UAVs.

\subsubsection{Power Line Detection-based Approaches}

A knowledge-based technique specifically designed for power line detection in aerial images was proposed in [22] by combining bottom-up and top-down line detection approaches. First, a filter based on Pulse Coupled Neural Network (PCNN) was applied to remove background. Then, straight lines were detected using the Hough transform. Finally, spurious linear objects were eliminated using the K-means clustering approach. 
A similar three-step approach was also used in [23] for detecting power lines. First, linear objects in a clustered background were enhanced by a double-sided filter. Then, the Radon transform was used to detect straight lines. Finally, a parallel lines constraint was used to recognize power lines. Tang Wen Yang et al. proposed a three-step approach for detecting overhead power lines from UAV video images [24]. First, video images are binarized using an adaptive thresholding approach. Next, the Hough transform was utilized to detect lines-candidates in the binary images. Then, a fuzzy C-means (FCM) clustering algorithm, which uses the length and the slope of the detected lines as a feature vector, was applied to discriminate power lines from the detected line candidates. Finally, spurious lines were removed by using the properties of power lines.

In [25], an improved version of a Bayesian classifier was proposed for power line detection. The traditional Bayesian classifier was enhanced by utilizing heuristic knowledge obtained by applying the Hough transform to determine the prior and posterior probabilities. The Bayesian classifier was also used in [26] for detecting power lines in images collected by helicopters. To begin with, the Hough transform was used to improve the Bayesian classifier for classifying pixels. Then, small components (misclassified pixels) were removed from the classified images by performing connected component analysis.

A novel method named Circle Based Search (CBS) for power line detection based on the search of lines between two opposite points was proposed in [27]. First, Canny and Steerable filters were utilized to segment power line images taken from UAVs. Then, the CBS method, which was based on geometric relationships, together with a procedure for connecting contiguous segments were used for detecting power lines from the segmented images.

A sequential local-to-global power line detection algorithm for detecting power lines from optical images was proposed in [28]. First, line segments with symmetrical edges were detected using a matched filter (MF) and first-order derivative of Gaussian (FDOG) to create an edge map. Next, non-power-line candidates were filtered out by using a morphological filter. Finally, a graph-cut model based on graph theory was proposed to group the detected line segments into whole power lines.

To address the threshold selection problem in traditional edge detection approaches as well as the Hough transform, Guang Zhou et al. proposed a method that can effectively tune the threshold in edge and line detection algorithms in [9]. A database was created for keeping optimal parameters for visited places, which can be retrieved later based on GPS coordinates. For unvisited places, an objective function and a power line model were used to predict the best parameters.

Jingjing Zhang et al. proposed a method for detecting and tracking power lines in complex environments [29]. First, line segments were extracted using the Hough transform. Second, an improved K-means algorithm in Hough space was employed to cluster the extracted line segments to detect power lines. Finally, the detected power lines were tracked by a Kalman filter in Hough space.

Michael Gerke et al. proposed a method for detecting and tracking power lines from cluttered backgrounds [30]. To begin with, a range filter was used to filter input images in four directions. Next, the images were converted to binary images, and morphological operations were then employed to remove unwanted objects. Then, the Canny edge detector and the Hough transform were used to detect power lines. Finally, the detected power lines were tracked with a gimbal system.

Dewi Jones et al. proposed a method for detecting and tracking power lines [31]. First, the contrast of input images were enhanced. Second, an edge map was formed by applying gradient computation and non-maximum suppression. Then, the Hough transform was computed thresholded followed by clustering points in the Hough transform to form the Aggregated Hough transform (AHT). Finally, tracking and acquisition were performed on the points in the AHT.

In summary, the presented approaches follow a general sixstage line detection/tracking process. First, input images are enhanced to remove noise. Second, background are removed using, for example, color-based background suppression and/or Pulse Coupled Neural Networks. Third, edge detectors, such as the Canny edge detector and steerable filters [27], are applied to generate edge maps. Fourth, straight lines from the generated edge maps are detected using, for instance, the Hough transform, the Radon transform, and/or Circle Based Search [27]. Fifth, clustering approaches (e.g., the K-means clustering and fuzzy C-mean clustering [24]) and/or power line constraints, such as parallel lines, are applied to eliminate spurious lines and detect power lines. Finally, the detected power lines are tracked using, for example, visual tracking methods based on fuzzy logic and Kalman filters. Although this general six-stage line detection/tracking system is simple to implement and has been widely used in power line inspection, it has many drawbacks, such as low speeds and inaccuracy. Thus, this approach is not well-suited for high-speed, fully autonomous vision-based navigation.

\subsubsection{Pole Detection-based Approaches}

An approach for identifying power pylons using UAVs and estimating the relative distance between the pylons and the UAVs was proposed in [32]. First, a line segment detector (LSD) algorithm was adopted to detect lines segments. Second, line segments belonging to the background were removed by a colour filter. Third, a triple matching strategy which uses Euclidean distance between the description histograms of the two segment candidates as metric and Left Right Checking (LRC) as criterion was proposed to match line segments across image sequences. Finally, an approach that integrates ego-motion of the UAV and the variation of the object size for estimating the depth of the pylons was proposed.

Ian Golightly and Dewi Jones proposed an approach for detecting and matching corners for visual tracking in power line inspection [33]. To begin with, a corner map that contains clusters of corners was produced by a Cooper, Venkatesh, Kitchen (CVK) corner detector. Then, singleton corner points were removed by cluster aggregation. Finally, a basic corner matcher was proposed to track the detected corners in image sequences.

An approach for detecting and tracking electric towers was proposed in [34]. First, a two-class multilayer perceptron 
(MLP) neural network was trained for tower-background classification based on HOG (Histogram of Oriented Gradients) features. Second, the network was applied as a sliding window detector. Third, a hierarchical tracking-by-registration tower tracker algorithm, which uses Hierarchical Multi-Parametric and Multi-Resolution Inverse Compositional, was propped to track the detected towers.

It has been shown in the last two sections that pole detectionbased approaches for navigation have been receiving much less attention compared to power line detection-based approaches. This is partly due to its main drawback which is the lack of information for navigating between poles. In conclusion, although many attempts have been made to improve visionbased navigation algorithms in the last decades, to the best of our knowledge, no high-speed, fully autonomous, vision-based navigation system for power line inspection has been successfully developed. Thus, we are in an urgent need for a novel vision-based navigation system which is not only fast but also accurate and capable of providing enough information for navigation between poles.

\subsection{Vision-based Approaches for Inspection}

As presented in section 3.2, there are four main inspection tasks that are well-suited for automatic autonomous visionbased inspection using UAVs: mapping and inspection of power line components, vegetation monitoring, icing detection and measurement, and disaster monitoring. In this section, we review some of the most recently published vision-based research approaches which have the potential in automating the above mentioned tasks.

\subsubsection{Mapping and Inspection of Power Line Components}

Wu Haibin et al. proposed an approach for detecting dampers in helicopter inspection of power transmission lines [35]. First, a threshold method was adopted to segment dampers from inspection images. Then, a modified version of the balloon force Snake method and Hessian matrix were utilized to detect dampers from the segmented images.

A novel system utilizing Faster R-CNN object detection framework and VGG16 model for detecting dead end body components (DEBC) was proposed in [36]. The authors proposed a series of simple image processing techniques for augmenting 146 input images to create 2437 training samples. The porposed data agumentation techniques include manually cropping, rotation, morphological operations (slight dilation and erosion), and "raster scan pattern" cropping. The system was tested on 111 aerial inspection photos, and achieved 83.7\% accuracy and $91 \%$ precision. The detection accuracy and precision were increased to $97.8 \%$ and $99.1 \%$ by adding 270 additional training images and including a new insulator class.

A vision-based approach for broken spacer detection was proposed in [37]. To begin with, the Canny edge detector, the Hough transform, and scanning window approach were utilized to detect spacers as region of interest (ROI). Second, image features were extracted using morphological operations. Finally, broken spacers were detected by connected domain analysis.
Wai Ho Li et al. proposed an image processing system for conductor localization and spacer detection [38]. To begin with, conductors were localized by applying a template matching approach in a sliding window fashion. Then, the conductor localization results were used to crop and rotate images. Finally, Gabor filters were applied on the cropped and rotated images to detect spacers by looking for large clusters of pixels that respond strongly to the filtering process.

An approach based on artificial neural networks (ANNs) for identifying poles and crossarms in images of power distribution lines was proposed in [39]. First, an ANN was trained to classify image pixels into four classes: poles, crossarms, vegetation, and others. Second, a threshold filter was used to remove unwanted areas. Finally, a template matching approach was applied to identify crossarms and poles. Carlos Sampedro et al. proposed an approach based on HOG features and MLP neural networks for detecting and classifying electric towers for power line inspection in [40]. First, a 2-class MLP was trained for background-foreground segmentation in a slidingwindow fashion. Then, a multi-class MLP was trained for classifying within four different types of electric towers. A fourstage approach for detecting transmission towers from aerial images was proposed in [41]. First, filtering via Optimized Mean Shift Segmentation was utilized to reduce background clutter and simultaneously accentuate the foreground. Second, candidate granules were selected based on gradient density and cluster density based thresholding. Third, the selected granules were merged via shared boundary criterion. Finally, context information was used to discard false positives. An approach for detecting electricity pylons in aerial video sequences was proposed in [42]. First, straight line pixel extraction was performed by combing a two-dimensional separable infinite impulse response (IIR) filter and non-maximal suppression. Next, straight lines were detected using the Hough transform. Finally, electricity pylon detection was performed based on the detected power lines. Xian Wang et al. proposed an approach for identifying insulators from aerial images in [43]. To begin with, a novel approach based on Otsu threshold and morphological operations was proposed for background suppression. Then, a Support Vector Machine (SVM) model was trained on features extracted by Gabor filters to classify insulators. In [44], a robust algorithm for detecting insulators in aerial images was proposed. First, an improved Harris corner selection strategy was used to detect local features. Second, a multiscale and multifeature (MSMF) descriptor was proposed to represent the local features. Third, several spatial orders features (SOFs) were found to improve the robustness of the algorithm. Finally, a coarse-tofine matching strategy was utilized to determine the region of insulators. Jingjing Zhao et al. proposed an approach based on lattice detection for detecting insulators in images of overhead transmission lines in [45]. First, corners detected by the KLT corner detection algorithm were used as low-level visual features. Mean shift clustering was then used to cluster the corners to find the repeating features that can represent the insulators. Next, insulators lattice modes were proposed based on a voting mechanism. Then, insulators were localized by performing lattice finding using a Markov Random Field (MRF) model to- 
gether with the spacial context information. Finally, minimum bounding rectangles of the target image was extracted by analyzing the geometric characteristics of the insulators region. An approach for detecting and inspecting insulator were proposed in [46]. In the detection phase, a part-based model with Circular GLOH-like (CGL) descriptor, which treats each insulator cap as one part of the model, was built. Next, a k-Nearest Neighbors $(\mathrm{kNN})$ classifier with the descriptors of detected Difference of Gaussians (DoG) keypoints was trained for distinguishing between insulators cap and clutter. Then, the bounding boxes for the insulators were determined from the classified keypoints. In the inspection phase, the detected insulators were first partitioned into caps. Then, a Elliptical GLOH-like (EGL) descriptor was extracted from each cap. Finally, Local Outlier Factor (LOF) approach was utilized to identify faulty caps.

A method for detecting cables damaged by lightning strokes was proposed in [47]. To begin with, the statistical analysis of the brightness of the cable, which is based on the mean brightness of the cable and its standard deviation, was performed to detect arc marks. Then, shape information, which was obtained from the comparison between a real cable contour and an ideal cable contour, was utilized for detecting cut wires.

\subsubsection{Vegetation Encroachment Monitoring}

A novel method for monitoring vegetation encroachment of transmissions lines using cameras integrated on each transmission pole was proposed in [48]. First, initial reference frames were acquired from the cameras. Next, image frames with inappropriate illumination (e.g., rainy images, foggy images) were filtered out. Then, the Hough transform was utilized to identify the horizontal and vertical lines of far away poles. Finally, motion tracking based on Laplacian kernel filtering and background-subtraction were applied to detect the encroached vegetation in the current scene images with respect to the reference images.

Juan I. Larrauri et al. developed an automatic inspection system based on UAVs for buildings, trees, and vegetation encroachment monitoring and for detecting bad conductivity and hot spots on power lines in [10]. First, power lines were identified and recognized based on a regularized Hough transform. Second, the distance between the UAVs and the power lines was calculated. Third, the distance between the conductor lines and the ground was calculated based on inputs from laser altimeters. Fourth, trees, vegetation, and buildings were detected based on the identification of solid surfaces or group of pixels that contain the same intensity value. Finally, stereoscopic vision methods using consecutive image frames were applied for calculating the distance from the conductor lines to trees, vegetation, and buildings.

\subsubsection{Icing Detection and Measurement}

Huang Huan et al. proposed an approach for measuring icing thickness of transmission lines based on photogrammetry method in [12]. To begin with, images of power lines were obtained by a high resolution camera. Next, the distance and level angle of a target below the power lines were recorded by a laser rangefinder and an IMU. Then, icing thickness parameter was calculated based on the recorded distance and angel.

A new image-based algorithm for icing detection and icing thickness estimation was proposed in [11]. First, edge detection accuracy was improved by using an ice-prior-based scheme. Next, a novel method for calibrating a monitoring camera based on single-image-based scheme was proposed. Then, a new thickness estimation scheme was proposed by introducing calibration information and utilizing a ROI tracking scheme.

\subsubsection{Disaster Monitoring}

Yan Liu et al. proposed an approach for estimating the height of power transmission towers with the single TerraSAR-X imagery in [49]. According to the authors, the proposed approach opened many possibilities for monitoring the situation of power transmission towers in natural disaster conditions since it is based on images from SAR, which is capable of producing high resolution radar images in all-weather conditions.

A review of some of the possible applications of UAVs for routine and emergency power line inspection was presented in [50]. According to the authors, damage assessments after extreme events, such as hurricanes, wind storms, and snow storms, is the most immediate application for UAVs. With the ability to access hard-to-reach areas and fly at high speed, UAVs allow almost immediate assessment of power line damage after natural disasters.

To conclude, mapping and inspection of power line components is the main focus of most of the previous studies, whereas vegetation monitoring, icing detection and measuring, and disaster monitoring have been receiving much less attention. Many task-specific, traditional vision-based approaches have been proposed to detect and inspect power components (e.g., spaces and dampers) and power lines. A few deep learning approaches, which are based on ANNs, multi-class MLPs, and CNN-based object detectors such as Faster-RCNN, have been applied for detecting and inspecting poles, crossarms, and dead end body components. Although these approaches work to some extend, they still have many drawbacks. In the next sections, we analyze the drawbacks in detail, and based on that, we propose a new automatic autonomous power line inspection concept.

\subsection{Limitations of Current Vision-based Inspection Systems}

It can be clearly seen from the review that even though many attempts have been made to automate power line visual inspection, no fully automatic autonomous vision-based inspection system that is capable of detecting a wide range of faults has been successfully developed. Most of the previous studies presented in the review focused on proposing task-specific approaches for either inspection or navigation. Those taskspecific approaches worked to some extend for tasks that they were designed for; however, they showed great limitations not only in terms of accuracy but also in terms of the ability to adapt to related tasks. Hence, a lot of efforts are required for developing a fully automatic autonomous vision-based inspection system since each sub-task (e.g., detecting poles for navi- 
gation, detecting broken insulators, detecting missing toppads) requires its own carefully hand-designed solution.

Thus, we are in an urgent need for a new approach that is (i) more accurate, (ii) requires less effort in hand-designing solution, (iii) and generalizes well across related tasks. To address the existing limitations of current vision-based inspection systems, we propose deep learning as a potential data analysis approach for moving toward automatic autonomous visionbased inspection because of the following reasons: First, deep learning algorithms, especially Convolutional Neural Networks (CNNs, or ConvNets), have greatly improved the performance of visual recognition systems for many advanced applications such as self-driving cars, image search, and image understanding. Second, deep learning provides a general method for automatically learning features, which can dramatically reduce the effort in hand-designing solutions for every sub task in power line inspection. Finally, deep learning approaches typically generalize well across related tasks [51]. The generalization ability of deep learning opens great possibilities for visionbased inspection since a model trained for one task with a very little effort in fine-tuning can be adapted for use in many other related tasks.

To facilitate the use of deep learning algorithms, particularly ConvNets, in addressing the existing problems of current vision-based inspection systems, in the next section, we review the potential role of deep learning and ConvNets as an advanced data analysis approach and as a potential solution to move forward automatic autonomous vision-based inspection.

\section{The Potential Role of Deep Learning for Automatic Au- tonomous Vision-based Power Line Inspection}

With the aim of utilizing recent advances in deep learning (DL) and UAV technologies for facilitating automatic autonomous UAV-based inspection of power line, eSmart Systems ${ }^{1}$ has initiated a project, code named Connected Drone. The project is expected to run from 2016 to at least until the end of 2018, and involves as of today 12 Norwegian power grid companies (such as Hafslund and Ringeriks-Kraft), universities (NORUT/ASUF, NTNU AMOS, and UiO), technology partners (Telenor, Microsoft, Teleplan Globe, and Eker Design), and drone experts (IRIS Group and Robot Aviation). The current work represented by this paper is funded by The Research Council of Norway (RCN) and eSmart Systems as an industrial $\mathrm{PhD}$ project in collaboration with the UiT Machine Learning Group $^{2}$. In this project, we have developed a system for automatically mapping and inspecting power line components using ConvNet-based object detection and classification models (SSD [52] and ResNet [53]). The system is capable of mapping basic power components including poles, toppads, crossarms, and insulators (Fig. 3) and inspecting common faults on the components, for instance incorrectly mounted insulators, cracked poles, and missing toppads (Fig. 4). This line of work has in

\footnotetext{
${ }^{1}$ eSmart Systems: https : //www . esmartsystems . com/

${ }^{2}$ UiT Machine Learning Group: http://site.uit.no/ml
}

our opinion demonstrated a potential promising role of deep learning, especially ConvNets, for automatic mapping and inspection of power line components, but has also revealed many challenges. From this starting point, we discuss in this section the potential role of deep learning and ConvNets in automatic autonomous vision-based power line inspection using UAVs based on the reviews presented in Section 4 and our own work.

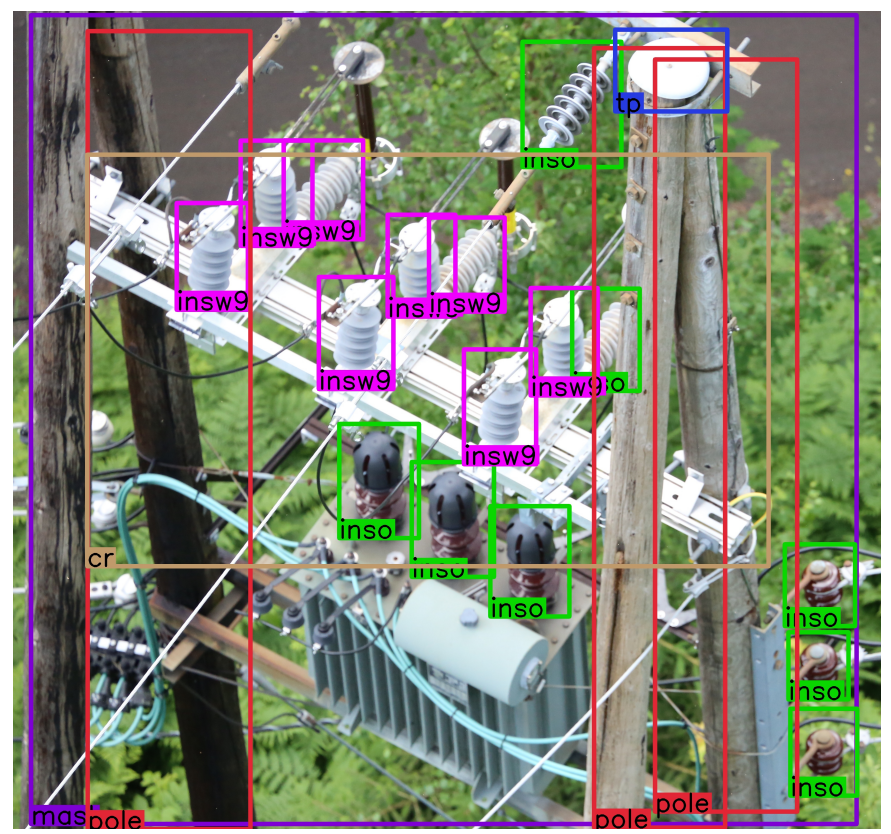

Figure 3: An illustration power line component mapping using our proposed power line inspection system. First, a power mast detection model is utilized to detect power masts from images acquired by UAVs. Then, the detected power masts are cropped from input images and passed through a power component detection model for mapping.

The remaining of the section is divided in three. First of all, we highlight the possibilities of DL vision-based navigation and DL vision-based inspection using UAVs. Secondly, we identify challenges of applying DL vision-based approaches in developing a fully automatic autonomous UAV-based inspection system. Finally, we suggest potential solutions to the challenges and propose approaches for facilitating the use of DL visionbased approaches in both UAV navigation and UAV inspection.

\subsection{Possibilities of DL for Vision-based UAV Inspection}

\subsubsection{Vision-based UAV Navigation}

DL vision-based navigation approaches (e.g., pole detectionbased and power line detection-based) can be combined with traditional navigation approaches, such as GPS way pointsbased, and UAV autopilots to facilitate self-driving UAVs. This not only can dramatically reduce the risk of power line inspection, but it also can increase the speed of online inspections and data acquisition for offline inspections.

In addition, DL vision-based approaches hold great promise for facilitating the implementation of automatic data acquisition (ADA) systems. In these systems, outputs from pole detectors 
and/or power line detectors are utilized for guiding cameras focus when taking pictures of power line components; as a result, the need of camera operators is eliminated and the quality of data acquisition is increased.

The combination of a self-driving UAV and an ADA system forms a basic automatic autonomous UAV-based power line inspection system. With a predefined flight plan, the UAV can automatically navigate along power lines to collect data for later offline inspections and perform online inspections.

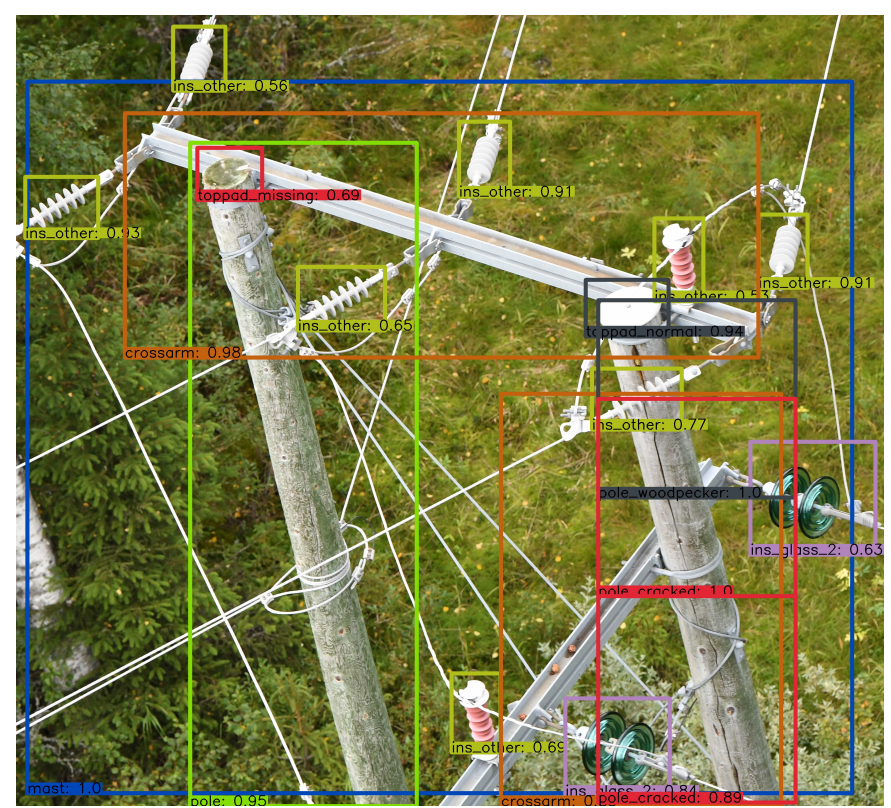

Figure 4: An illustration of power line component inspection using our proposed power line inspection system. Results produced by power line component mapping models (Fig. 3) are passed through fault detection models to detect potential faults, such as missing toppads (toppad_missing), incorrectly mounted insulators (insb4_side), cracked poles (pole_cracked), and woodpecker attacks (pole_woodpecker).

\subsubsection{Vision-based UAV Inspection}

Advanced DL vision-based approaches and UAVs technologies offer many possibilities for automating the four most common tasks in power line inspection: mapping and inspection of power line components, icing Detection and measurement, vegetation encroachment monitoring, and disaster monitoring. For example, state-of-the-art object detectors powered by deep convolution neural networks (e.g., Faster R-CNN [54], SSD [52], YOLO [55], and R-FCN [56]) together with deep neural networks for image classification, such as ResNet [53], Inceptionv4, and Inception-ResNet [57], can be used for detecting, classifying, and mapping power line components (Fig. 3). Next, deep learning-based semantic segmentation approaches (e.g., DPN [58] and Mask R-CNN [59]) and/or traditional background removal methods, for example colour-based suppression [60] and pulse coupled neural filter (PCNF) [22], can be utilized for removing background from the detected components. Finally, inspections can be performed on the segmented images by, for example, performing texture analysis and/or vision-based anomaly detection to detect faults (Fig. 2). The above mentioned approaches are also useful in the case of disaster monitoring. For instance, a tree detection model can be used together with a power line detection model in detecting trees lying across or against power lines after natural disasters, such as storms, hurricanes, and earthquakes.

In the case of vegetation encroachment monitoring and icing detection and measurement, detecting power lines is one of the most challenging tasks. However, recent advances in deep vision have opened up many possibilities for addressing this challenge. For example, state-of-the-art deep CNN-based edge detectors, such as Holistically-Nested Edge Detection [61], and contour detectors, for instance DeepEdge [62] and DeepContour [63], can be applied to produce very high quality edge maps. The edge maps can then be used by traditional straight line detection methods (e.g., Hough transform [64]) to detect power lines.

\subsection{Challenges of DL Vision-based UAV Inspection}

\subsubsection{Vision-based UAV Navigation}

According to [9], there are three common approaches for UAV navigation in automatic power line inspection: pole detection-based, GPS way points-based, and power line detection-based. Unfortunately, none of the three methods offer a sufficient navigation accuracy for fully automatic autonomous UAV inspection.

Pole detection-based navigation detects electricity poles without providing specific methods for navigating along the power lines. In addition, when multiple poles are detected, it is very challenging to correctly identify the next target pole. In contrast, GPS way points-based navigation has no problem identifying the next target pole; nevertheless, it requires prespecified exact locations of every electricity poles, which is typically not available for many existing power grids. Furthermore, GPS has a large error range that could result in low navigation accuracy. The power line detection-based navigation provides sufficient information for UAVs to navigate along the power lines; however, power lines are typically very thin and lack of rich features. Thus, detecting and tracking the lines is an extremely challenging task. Another major challenge of the power line detection-based approach is "out-of-sight navigation". During inspection, UAVs might be blew far away from power lines by, for example, strong wind. This could result in a deadlock since UAVs no longer "see" the power lines.

\subsubsection{Vision-based UAV Inspection}

Our work in automating UAV-based power line inspection has in our opinion demonstrated the potential role of deep learning for automatic mapping and inspection of power line, but has also revealed many challenges.

The first challenge is the lack of training data. Deep learning models for mapping and inspection of power line components typically require a huge amount of data to train. Unfortunately, to the best of our knowledge, there are no publicly available datasets that are big enough for training such models. The most straightforward solution is to create a training dataset from scratch by manually tagging images; however, it is a very slow, 
tedious, and expensive process. To move forward, we have created a medium-sized dataset by manually tagging 30000 images with 54 classes (e.g., toppad_plastic, toppad_metal, pole, transformer). The average number of objects per image was 8 ; the average tagging speed (for a normal person) was 40 images per hour; thus, it required around 750 working hours to create the dataset.

The second challenge comes from the long-tailed distribution of component classes, which is also known as the class imbalance problem [65]. Datasets for training object detection, image classification, and semantic segmentation models are typically required to have balanced classes. In other words, all classes in an ideal training dataset should have a similar number of examples [66]. Unfortunately, in power line images, a small number of component classes, such as Insulator-Brown-4 and WoodenPole, appear very often while most of the other classes, for instance Broken-Insulators and Toppad-Missing, appear more rarely. For example, in the 30000-image dataset that we created, there are 43275 examples of the Insulator-Brown-4 class while only 210 examples of the Toppad-Missing class are found (Fig. 5). The imbalance of object classes typically makes deep learning models bias towards classes that have more examples and overlook classes which have fewer examples. This is a huge problem in power line inspection since the classes which have very few examples are usually the faults that we need to identify, such as missing toppads, broken insulators, and cracked poles.

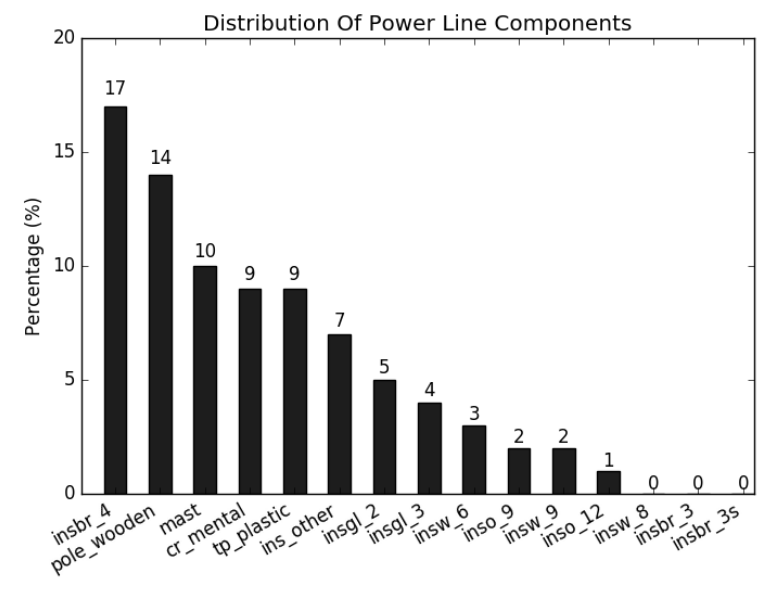

Figure 5: Distribution of the 15 most common power line component classes in our 30000-image dataset. While there are 43275 (17\%) examples of the Insulator-Brown-4 (insbr_4) class, only 210 (0.08\%) examples of the ToppadMissing (tp_missing) class are found.

The third challenge is the detection of small power components and small faults. Most of the well-known CNN-based object detectors, such as SSD [52], YOLO [55], R-FCN [56], and Faster R-CNN [54], perform poorly on very small objects [67]. This is a major problem in mapping and inspection of power line components because many important power components (e.g., insulators) and faults on power lines, for instance missing toppads, broken wires, and broken insulators, are very small compared to other components (e.g., poles, crossarms) and surrounding objects, such as trees (Fig. 6).
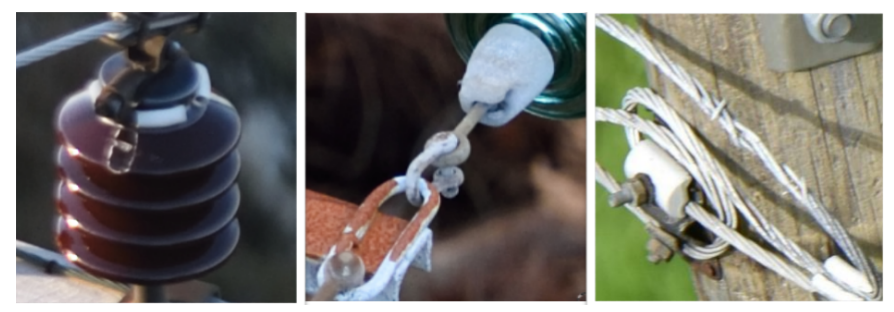

Figure 6: Small faults on power lines components (from left to right): cracked insulator, missing splint, broken wire.

The fourth challenge is the detection of unseen components and faults. Many existing power grids, especially in Norway, utilize a wide range of power components; thus, the grids are vulnerable to a huge number of types of faults. In addition, faults on power components and power lines, even simple ones such as missing toppads, can occur in many different forms (Fig. 7). This, together with the lack of training data, pose a major challenge for DL vision-based inspection of power line due to the limitation of deep learning models in predicting previously unseen classes.
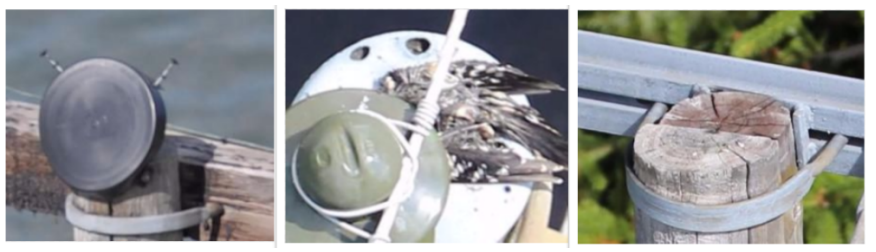

Figure 7: Faults on toppads can be in many different forms (left to right): about to be missing, covered by unwanted objects, missing.

The fifth challenge is the detection of power lines in cluttered backgrounds. In both UAV navigation and UAV inspection (icing detection and measurement, vegetation encroachment monitoring), detecting power lines is one of the most crucial task. However, this is a very challenging task for two reasons: First, power lines in images taken from UAVs or helicopters are very thin leading to a lack of rich features for their representation. Second, because of color similarity, weather conditions, and lightning conditions power lines are typically very difficult to be separated from backgrounds. For example. during the winter in cold countries, such as Norway, both power lines and the ground are usually covered in snow and/or ice making them indistinguishable, even for humans (Fig. 1).

The last but not least challenge is the lack of metrics in evaluating the performance of DL vision-based UAV inspection systems. Since there are no publicly available datasets for power line inspection that have a significant number of examples of faults, how to evaluate the performance of DL vision-based UAV inspection systems still remained an unsolved problem. Synthetic images of faults can be used to address the challenge to some degree; however, the performance of the system on synthetic images typically does not give an accurate estimation of the performance of the system on real images. 


\subsection{Possible Solutions}

\subsubsection{Vision-based UAV Navigation}

A potential solution for UAV navigation in automatic autonomous power line inspection is to combine the GPS way points-based, pole detection-based, and power line detectionbased navigation approaches with an autopilot to build a hybrid navigation system. In this system, outputs from a pole detector can be used to together with GPS way points to accurately identify the next target poles. Based on that, an autopilot can be utilized to navigate the UAV to the identified poles by, for example, following the lines detected by a power line detector and/or tracker.

To address the "out-of-sight navigation" problem, wide angel cameras, such as 360-degree cameras, can be applied to ensure that UAVs can always "see" the power lines during navigation.

\subsubsection{Vision-based UAV Inspection}

There are four main approaches for mapping and inspection of power line components. The first approach is based on the comparison of power masts with their ideal models. This is a relatively simple approach; however, it is required that the ideal models must contain the perfect spatial configuration of the power masts, which is typically quite tedious, time-consuming, and expensive to create. The second method is related to the detection of changes that appear after the last inspection. For example, the structure of a power mast, which is usually defined by its components and their relative position, can be estimated and compared with results from the previous inspection to detect changes, which may lead to power outages, for instance missing splints, missing toppads, and missing insulators. Similar approach can be applied for the detection of differences among a set of neighbouring pylons of the same type, usually in the same power line. Neighboring pylons of the same type in the same power line typically have the same architecture except for pylons at special locations; thus, faults on a power line can be detected by measuring the consistency of power mast structure on that line [68]. The final approach is related to the direct detection of faults from inspection images, usually based on deep learning models and/or traditional vision-based approaches, such as texture analysis and pattern matching. Each of the above-mentioned approaches has its own advantages and comes with unique challenges. Following are our proposed solutions to some of the major challenges.

One of the most straightforward approaches for dealing with the lack of training data problem is to manually create training data; however, this is a very slow, tedious, and expensive process. A potential way to speed up the process is to use pretrained models and fine-tune them with a small amount of manually created training data to automatically create more data.

When only a small amount of training data is available, data augmentation techniques can be utilized to increase training performance. Some examples of simple data augmentation techniques that are useful for training deep learning models are flipping, cropping, and color jittering. Recent advances in image style transfer have opened up new possibilities in advanced data augmentation by, for example, transferring the time of day, weather, and season [69, 70, 71].
Another solution to the lack of training data problem is to use synthetic images. However, how to effectively combine synthetic images with real images in training deep learning models still a challenging question. To address this challenge to some extend, supervised domain adaptation [72] can be applied. In this approach, a model is first trained only on synthetic images and/or images from related tasks; it is then fine tuned for the target task that typically has very few training examples. In the case of no training examples available, unsupervised domain adaptation [73, 74, 75, 76], which is capable of adapting models trained only on synthetic images and/or images from related tasks to use on the target task, is a potential solution.

The class imbalance problem can be tackled to some extent with synthetic images by, for example, generating more synthetic images for classes that have fewer training samples to balance out the imbalanced classes. Another alternative solution is to use median frequency balancing approach in which classes with fewer training examples will be assigned higher weights during training $[77,78,79]$.

To detect small components and faults, object detection, image classification, and semantic segmentation pipelines can be utilized. For example, a power mast detection model can be applied to locate masts in inspection images and crop them as Region of Interests (ROIs). Then, detailed component and fault detection models can be employed to detect small components (e.g., insulators, toppads) and faults (missing toppads, cracked poles) from the cropped ROIs. The detected components can be further cropped and used as inputs for more detailed fault detection models to detect smaller faults, for example missing splints, broken wires, and cracked insulators (Fig. 6).

To detect unseen components and faults, one-shot learning [80, 81, 82, 83], which allows a trained model to learn to detect new classes (components and faults) from only one or a few examples per class, is a very promising approach. An alternative solution is to first train a model with synthetic images of components and faults, then adapt it for detecting real components and faults using unsupervised domain adaptation [73, 74, 75, 76] . Recently, advances in Generative Adversarial Networks (GANs) [84] have opened new possibilities for unsupervised anomaly detection. GANs can be employed for learning the data distribution that generates normal components. Then, different metrics, such as discrimination score and residual score, can be combined and used as "anomaly score" to perform anomaly detection [85]. The main advantage of this approach is that the training requires only images of normal components, which are relatively easy to collect.

In vegetation encroachment monitoring and icing detection and measurement, a power line detection pipeline can be utilized to address the thin line and the lack of rich feature challenges. First, outputs from edge detection algorithms, for instance the Canny edge detector [86], Matched filter [87], and Holistically-Nested Edge Detection [61], contour detectors such as, DeepEdge [62] and DeepContour [63], and/or line detectors, for example the Hough transform [64] and the Radon transform [88], can be used together with prior knowledge of power lines properties (e.g., parallel lines) to locate ROIs in low resolution images. Then, the identified ROIs are mapped 
to and cropped from higher resolution images. Finally, more advanced line detectors algorithms can be employed to detect power lines from the cropped ROIs in which power lines are typically bigger and have richer features than those in original images.

To separate power lines from clustered backgrounds, background removal approaches can be used as a pre-progressing step prior to the edge detection and line detection steps. Some examples of background removal techniques are colour based suppression [60], pulse coupled neural filter (PCNF) [22], and deep learning-based semantic segmentation (e.g., DPN [58] and Mask R-CNN [59]). After the background is removed, clustering approaches (e.g., the K-means clustering [89] and fuzzy C-means clustering [90]) together with power line constrains (e.g., parallel lines) can be combined to eliminate spurious linear objects and detect power lines.

\subsection{Multimodal Inspection}

Optical image based fault detectors are capable of detecting a wide range of visual faults, for example missing toppads, pole cracks, and woodpecker attacks. However, they have numerous drawbacks, such as their sensitivity to illumination changes [91] and their incapability of detecting faults that are invisible to the unaided human eye (e.g., hot spots on power line components). To overcome the drawbacks, information of a visible camera can be fused with information provided by other sensors, such as thermal cameras and ultraviolet cameras, to perform multimodal inspection [92].

Recently, deep learning has been successfully used for learning from multimodal data sources for various vision tasks, for instance polar bear detection [93] and pedestrian detection $[91,94,95]$. This approach can be easily adapted to fuse images from multiple sensors, such as optical cameras, thermal cameras, and ultraviolet cameras, to improve the performance of power line inspection systems. The fusion can typically take place at three different levels of abstraction: pixel-level, feature-level, and decision-level fusion [91].

In power line inspection, images from different sensors are suitable for detecting a different set of faults. Optical images, for example, are well-suited for detecting visual faults, whereas thermal images and ultraviolet images are useful for detecting faults that are invisible to the unaided human eye, such as equipment bad connections and corona discharges. Thus, fusing images from multiple sensors can extend the range of faults that inspection systems can detect. In addition, images chanels fusion can also improve inspection performance since images chanels such as optical images and thermal images typically provide complementary visual information which are useful for deep learning models [94].

\section{Conclusion}

In this paper, we have presented a thorough literature review of automatic power line inspection research including vision-based approaches for both UAV navigation (power line detection-based and pole detection-based approaches) and UAV inspection (mapping and inspection of power line components, vegetation encroachment monitoring, icing detection and measurement, and disaster monitoring).

Further, we have summarized the possibilities of DL visionbased approaches and UAVs in developing a fully automatic autonomous power line inspection system.

Following a comprehensive review of current vision-based automatic power line inspection research approaches, we have identified existing challenges of both DL vision-based navigation and DL vision-based inspection and discussed solutions to these challenges.

Finally, with the aim of providing an initial starting point for researchers who are interested in developing a fully automatic autonomous vision-based power line inspection system using UAVs, we have proposed four potential next steps: (i) combine pole detection-based, GPS way points-based, and power line detection-based navigation approaches with autopilots to facilitate self-driving UAVs and automatic data acquisition, (ii) utilize multistage object detection, classification, and segmentation pipelines to detect faults in various sizes, forms, and conditions, (iii) employ contextual information (e.g., power mast structure) to further improve fault detection performance by, for example, eliminating invalid faults, and (iv) apply multiple data sources fusion (e.g., infrared cameras, ultraviolet cameras, and 3D cameras) for detecting complicated faults (e.g., cracked insulators, rotten poles, and corona discharges).

\section{Acknowledgment}

The authors would like to thank eSmart System AS (Dang $\mathrm{Ha}$ The Hien and Huyen Thi Phuong Vu) for support in the work with this paper. This work was supported by the Research Council of Norway [RCN NRINGSPHD grant no. 263894 (2016-2018) on Power Grid Image Analysis] and eSmart Systems AS.

\section{References}

[1] M. Bruch, V. Münch, M. Aichinger, M. Kuhn, M. Weymann, G. Schmid, Power blackout risks, in: CRO Forum, 2011, p. 28.

[2] A. Castillo, Risk analysis and management in power outage and restoration: A literature survey, Electric Power Systems Research 107 (2014) 9-15.

[3] Y. Pradeep, S. A. Khaparde, R. K. Joshi, High level event ontology for multiarea power system, IEEE Transactions on Smart Grid 3 (1) (2012) 193-202.

[4] L. Matikainen, M. Lehtomäki, E. Ahokas, J. Hyyppä, M. Karjalainen, A. Jaakkola, A. Kukko, T. Heinonen, Remote sensing methods for power line corridor surveys, ISPRS Journal of Photogrammetry and Remote Sensing 119 (2016) 10-31.

[5] J. Katrasnik, F. Pernus, B. Likar, A survey of mobile robots for distribution power line inspection, IEEE Transactions on Power Delivery 25 (1) (2010) 485-493.

[6] J. Ahmad, A. S. Malik, L. Xia, N. Ashikin, Vegetation encroachment monitoring for transmission lines right-of-ways: A survey, Electric Power Systems Research 95 (2013) 339-352.

[7] F. Mirallès, N. Pouliot, S. Montambault, State-of-the-art review of computer vision for the management of power transmission lines, in: International Conference on Applied Robotics for the Power Industry (CARPI), IEEE, 2014, pp. 1-6. 
[8] L. F. Luque-Vega, B. Castillo-Toledo, A. Loukianov, L. E. GonzalezJimenez, Power line inspection via an unmanned aerial system based on the quadrotor helicopter, in: MELECON 2014-2014 17th IEEE Mediterranean Electrotechnical Conference, IEEE, 2014, pp. 393-397.

[9] G. Zhou, J. Yuan, I.-L. Yen, F. Bastani, Robust real-time uav based power line detection and tracking, in: IEEE International Conference on Image Processing (ICIP), IEEE, 2016, pp. 744-748.

[10] J. I. Larrauri, G. Sorrosal, M. González, Automatic system for overhead power line inspection using an unmanned aerial vehiclerelifo project, in: International Conference on Unmanned Aircraft Systems (ICUAS), IEEE, 2013, pp. 244-252.

[11] Y.-p. Zhong, Q. Zuo, Y. Zhou, C. Zhang, A new image-based algorithm for icing detection and icing thickness estimation for transmission lines, in: IEEE International Conference on Multimedia and Expo Workshops (ICMEW), IEEE, 2013, pp. 1-6.

[12] H. Huang, X. Ma, L. Zhao, H. Du, H. Luo, X. Mao, M. Tang, Y. Liu, Transmission line icing measurement on photogrammetry method, in: 9th International Symposium on Multispectral Image Processing and Pattern Recognition (MIPPR2015), International Society for Optics and Photonics, 2015, pp. 98150Z-98150Z.

[13] A. Moreira, P. Prats-Iraola, M. Younis, G. Krieger, I. Hajnsek, K. P. Papathanassiou, A tutorial on synthetic aperture radar, IEEE Geoscience and remote sensing magazine 1 (1) (2013) 6-43.

[14] J. M. Lloyd, Thermal imaging systems, Springer Science \& Business Media, 2013

[15] A. Richards, O. P. Partner, Reflected ultraviolet imaging for forensics applications, Online] http://www. uvcorder. com/pdf/Reflected_UV_Imaging_for_Forensics_V2.pdf

[16] L. Chen, L. Lin, M. Tian, X. Bian, L. Wang, Z. Guan, The ultraviolet detection of corona discharge in power transmission lines, Energy and Power Engineering 5 (04) (2013) 1298.

[17] A. Wehr, U. Lohr, Airborne laser scanningan introduction and overview, ISPRS Journal of photogrammetry and remote sensing 54 (2) (1999) 6882.

[18] J. Beraldin, F. Blais, U. Lohr, Laser scanning technology, Airborne and terrestrial laser scanning (2010) 1-42.

[19] C. V. Tao, J. Li, Advances in mobile mapping technology, Vol. 4, CRC Press, 2007.

[20] A. Savvaris, Y. Xie, K. Malandrakis, M. Lopez, A. Tsourdos, Development of a fuel cell hybrid-powered unmanned aerial vehicle, in: 24th Mediterranean Conference on Control and Automation (MED), IEEE, 2016, pp. 1242-1247.

[21] T. Zhang, Q. Li, C.-s. Zhang, H.-w. Liang, P. Li, T.-m. Wang, S. Li, Y.-1. $\mathrm{Zhu}, \mathrm{C}$. Wu, Current trends in the development of intelligent unmanned autonomous systems, Frontiers of Information Technology \& Electronic Engineering 18 (1) (2017) 68-85.

[22] Z. Li, Y. Liu, R. Hayward, J. Zhang, J. Cai, Knowledge-based power line detection for uav surveillance and inspection systems, in: 23rd International Conference Image and Vision Computing New Zealand, IEEE, 2008, pp. 1-6.

[23] L. Zhu, W. Cao, J. Han, Y. Du, A double-side filter based power line recognition method for uav vision system, in: IEEE International Conference on Robotics and Biomimetics (ROBIO), IEEE, 2013, pp. 26552660 .

[24] T. W. Yang, H. Yin, Q. Q. Ruan, J. Da Han, J. T. Qi, Q. Yong, Z. T. Wang, Z. Q. Sun, Overhead power line detection from uav video images, in: 19th International Conference Mechatronics and Machine Vision in Practice (M2VIP), IEEE, 2012, pp. 74-79.

[25] S. Du, B. J. Van Wyk, C. Tu, Heuristic bayesian pixel classification for power line inspection, in: 3rd International Congress on Image and Signal Processing (CISP), Vol. 2, IEEE, 2010, pp. 960-963.

[26] Z. Gaspar, B. Shengzhi Du, Hough transform tuned bayesian classifier for overhead power line inspection, in: Proceedings of the 19th Annual Symposium of the Pattern Recognition Association of South Africa, 2008, pp. 137-140.

[27] A. Cerón, F. Prieto, et al., Power line detection using a circle based search with uav images, in: International Conference on Unmanned Aircraft Systems (ICUAS), IEEE, 2014, pp. 632-639.

[28] B. Song, X. Li, Power line detection from optical images, Neurocomputing 129 (2014) 350-361.

[29] J. Zhang, L. Liu, B. Wang, X. Chen, Q. Wang, T. Zheng, High speed au- tomatic power line detection and tracking for a uav-based inspection, in: International Conference on Industrial Control and Electronics Engineering (ICICEE), IEEE, 2012, pp. 266-269.

[30] M. Gerke, P. Seibold, Visual inspection of power lines by uas, in: International Conference and Exposition on Electrical and Power Engineering (EPE), IEEE, 2014, pp. 1077-1082.

[31] D. Jones, I. Golightly, J. Roberts, K. Usher, Modeling and control of a robotic power line inspection vehicle, in: Proceedings of the 2006 IEEE International Conference on Control Applications, IEEE, 2006, pp. 632637.

[32] O. Araar, N. Aouf, J. L. V. Dietz, Power pylon detection and monocular depth estimation from inspection uavs, Industrial Robot: An International Journal 42 (3) (2015) 200-213.

[33] I. Golightly, D. Jones, Corner detection and matching for visual tracking during power line inspection, Image and Vision Computing 21 (9) (2003) 827-840.

[34] C. Martinez, C. Sampedro, A. Chauhan, P. Campoy, Towards autonomous detection and tracking of electric towers for aerial power line inspection, in: International Conference on Unmanned Aircraft Systems (ICUAS), IEEE, 2014, pp. 284-295.

[35] W. Haibin, X. Yanping, F. Weimin, S. Xiaoming, J. Li, Damper detection in helicopter inspection of power transmission line, in: Instrumentation and Measurement, Computer, Communication and Control (IMCCC), 2014 Fourth International Conference on, IEEE, 2014, pp. 628632

[36] I. E. Nordeng, A. Hasan, D. Olsen, J. Neubert, Debc detection with deep learning, in: Scandinavian Conference on Image Analysis, Springer, 2017, pp. 248-259.

[37] Y. Song, L. Wang, Y. Jiang, H. Wang, W. Jiang, C. Wang, J. Chu, D. Han, A vision-based method for the broken spacer detection, in: IEEE International Conference on Cyber Technology in Automation, Control, and Intelligent Systems (CYBER), IEEE, 2015, pp. 715-719.

[38] W. H. Li, A. Tajbakhsh, C. Rathbone, Y. Vashishtha, Image processing to automate condition assessment of overhead line components, in: 1st International Conference on Applied Robotics for the Power Industry (CARPI), IEEE, 2010, pp. 1-6.

[39] P. B. Castellucci, L. C. Lucca, M. SantAnna, G. Traballe, V. H. Mustacio, J. F. R. da Silva, S. Vallin, Pole and crossarm identification in distribution power line images, in: Robotics Symposium and Competition (LARS/LARC), 2013 Latin American, IEEE, 2013, pp. 2-7.

[40] C. Sampedro, C. Martinez, A. Chauhan, P. Campoy, A supervised approach to electric tower detection and classification for power line inspection, in: International Joint Conference on Neural Networks (IJCNN), IEEE, 2014, pp. 1970-1977.

[41] T. Dutta, H. Sharma, A. Vellaiappan, P. Balamuralidhar, Image analysisbased automatic detection of transmission towers using aerial imagery, in: Iberian Conference on Pattern Recognition and Image Analysis, Springer, 2015, pp. 641-651.

[42] J. Tilawat, N. Theera-Umpon, S. Auephanwiriyakul, Automatic detection of electricity pylons in aerial video sequences, in: International Conference on Electronics and Information Engineering (ICEIE), Vol. 1, IEEE, 2010, pp. V1-342.

[43] X. Wang, Y. Zhang, Insulator identification from aerial images using support vector machine with background suppression, in: International Conference on Unmanned Aircraft Systems (ICUAS), IEEE, 2016, pp. 892 897

[44] S. Liao, J. An, A robust insulator detection algorithm based on local features and spatial orders for aerial images, IEEE Geoscience and Remote Sensing Letters 12 (5) (2015) 963-967.

[45] J. Zhao, X. Liu, J. Sun, L. Lei, Detecting insulators in the image of overhead transmission lines, in: International Conference on Intelligent Computing, Springer, 2012, pp. 442-450.

[46] M. Oberweger, A. Wendel, H. Bischof, Visual recognition and fault detection for power line insulators, in: 19th Proceedings of Computer Vision Winter Workshop (CVWW), 2014, pp. 81-88.

[47] R. Ishino, F. Tsutsumi, Detection system of damaged cables using video obtained from an aerial inspection of transmission lines, in: Power Engineering Society General Meeting, IEEE, 2004, pp. 1857-1862.

[48] J. Ahmad, A. S. Malik, M. F. Abdullah, N. Kamel, L. Xia, A novel method for vegetation encroachment monitoring of transmission lines using a single 2d camera, Pattern Analysis and Applications 18 (2) (2015) 419-440. 
[49] L. Yan, W. Wu, T. Li, Power transmission tower monitoring technology based on terrasar-x products, in: International Symposium on Lidar and Radar Mapping Technologies, International Society for Optics and Photonics, 2011, pp. 82861E-82861E.

[50] J. Toth, A. Gilpin-Jackson, Smart view for a smart gridunmanned aerial vehicles for transmission lines, in: 1st International Conference on Applied Robotics for the Power Industry (CARPI), IEEE, 2010, pp. 1-6.

[51] Y. LeCun, Y. Bengio, G. Hinton, Deep learning, Nature 521 (7553) (2015) 436-444.

[52] W. Liu, D. Anguelov, D. Erhan, C. Szegedy, S. Reed, C.-Y. Fu, A. C. Berg, Ssd: Single shot multibox detector, in: European conference on computer vision, Springer, 2016, pp. 21-37.

[53] K. He, X. Zhang, S. Ren, J. Sun, Deep residual learning for image recognition, in: Proceedings of the IEEE Conference on Computer Vision and Pattern Recognition, 2016, pp. 770-778.

[54] S. Ren, K. He, R. Girshick, J. Sun, Faster r-cnn: Towards real-time object detection with region proposal networks, in: Advances in neural information processing systems, 2015, pp. 91-99.

55] J. Redmon, S. Divvala, R. Girshick, A. Farhadi, You only look once: Unified, real-time object detection, in: Proceedings of the IEEE Conference on Computer Vision and Pattern Recognition, 2016, pp. 779-788.

[56] J. Dai, Y. Li, K. He, J. Sun, R-fen: Object detection via region-based fully convolutional networks, in: Advances in neural information processing systems, 2016, pp. 379-387.

[57] C. Szegedy, S. Ioffe, V. Vanhoucke, A. Alemi, Inception-v4, inceptionresnet and the impact of residual connections on learning, arXiv preprint arXiv:1602.07261.

[58] Z. Liu, X. Li, P. Luo, C.-C. Loy, X. Tang, Semantic image segmentation via deep parsing network, in: Proceedings of the IEEE International Conference on Computer Vision, 2015, pp. 1377-1385.

[59] K. He, G. Gkioxari, P. Dollár, R. Girshick, Mask r-cnn, arXiv preprint arXiv:1703.06870.

[60] M. Rajeev, V. Adithya, S. Hrishikesh, P. Balamurali, Detection of powerlines in complex natural surroundings, Computer Science \& Information Technology (CS \& IT) Volume 3 (2013) 101-108.

[61] S. Xie, Z. Tu, Holistically-nested edge detection, in: Proceedings of the IEEE International Conference on Computer Vision, 2015, pp. 13951403.

[62] G. Bertasius, J. Shi, L. Torresani, Deepedge: A multi-scale bifurcated deep network for top-down contour detection, in: Proceedings of the IEEE Conference on Computer Vision and Pattern Recognition, 2015, pp. $4380-4389$

[63] W. Shen, X. Wang, Y. Wang, X. Bai, Z. Zhang, Deepcontour: A deep convolutional feature learned by positive-sharing loss for contour detection, in: Proceedings of the IEEE Conference on Computer Vision and Pattern Recognition, 2015, pp. 3982-3991.

[64] R. O. Duda, P. E. Hart, Use of the hough transformation to detect lines and curves in pictures, Communications of the ACM 15 (1) (1972) 11-15.

[65] B. Krawczyk, Learning from imbalanced data: open challenges and future directions, Progress in Artificial Intelligence 5 (4) (2016) 221-232.

[66] W. Ouyang, X. Wang, C. Zhang, X. Yang, Factors in finetuning deep model for object detection with long-tail distribution, in: Proceedings of the IEEE Conference on Computer Vision and Pattern Recognition, 2016, pp. 864-873.

[67] J. Huang, V. Rathod, C. Sun, M. Zhu, A. Korattikara, A. Fathi, I. Fischer, Z. Wojna, Y. Song, S. Guadarrama, et al., Speed/accuracy trade-offs for modern convolutional object detectors, arXiv preprint arXiv:1611.10012.

[68] P. Mazurek, K. Okarma, Application of background estimation and removal techniques for the extraction of the power line components on the digital images for the automatic power line inspection systems, Pomiary Automatyka Kontrola 54 (2008) 698-699.

[69] F. Luan, S. Paris, E. Shechtman, K. Bala, Deep photo style transfer, arXiv preprint arXiv:1703.07511.

[70] L. A. Gatys, A. S. Ecker, M. Bethge, Image style transfer using convolutional neural networks, in: Proceedings of the IEEE Conference on Computer Vision and Pattern Recognition, 2016, pp. 2414-2423.

[71] J.-Y. Zhu, T. Park, P. Isola, A. A. Efros, Unpaired image-to-image translation using cycle-consistent adversarial networks, arXiv preprint arXiv: 1703.10593.

[72] G. Csurka, Domain adaptation for visual applications: A comprehensive survey, arXiv preprint arXiv:1702.05374.
[73] Y. Ganin, V. Lempitsky, Unsupervised domain adaptation by backpropagation, arXiv preprint arXiv:1409.7495.

[74] M. Long, H. Zhu, J. Wang, M. I. Jordan, Unsupervised domain adaptation with residual transfer networks, in: Advances in Neural Information Processing Systems, 2016, pp. 136-144.

[75] M. Long, Y. Cao, J. Wang, M. I. Jordan, Learning transferable features with deep adaptation networks., in: ICML, 2015, pp. 97-105.

[76] O. Sener, H. O. Song, A. Saxena, S. Savarese, Learning transferrable representations for unsupervised domain adaptation, in: Advances in Neural Information Processing Systems, 2016, pp. 2110-2118.

[77] D. Eigen, R. Fergus, Predicting depth, surface normals and semantic labels with a common multi-scale convolutional architecture, in: Proceedings of the IEEE International Conference on Computer Vision, 2015, pp. 2650-2658.

[78] V. Badrinarayanan, A. Kendall, R. Cipolla, Segnet: A deep convolutional encoder-decoder architecture for image segmentation, arXiv preprint arXiv:1511.00561.

[79] M. Kampffmeyer, A.-B. Salberg, R. Jenssen, Semantic segmentation of small objects and modeling of uncertainty in urban remote sensing images using deep convolutional neural networks, in: Proceedings of the IEEE Conference on Computer Vision and Pattern Recognition Workshops, 2016, pp. 1-9.

[80] L. Fei-Fei, R. Fergus, P. Perona, One-shot learning of object categories, IEEE transactions on pattern analysis and machine intelligence 28 (4) (2006) 594-611.

[81] A. Santoro, S. Bartunov, M. Botvinick, D. Wierstra, T. Lillicrap, Oneshot learning with memory-augmented neural networks, arXiv preprint arXiv: 1605.06065 .

[82] O. Vinyals, C. Blundell, T. Lillicrap, D. Wierstra, et al., Matching networks for one shot learning, in: Advances in Neural Information Processing Systems, 2016, pp. 3630-3638.

[83] A. Mehrotra, A. Dukkipati, Generative adversarial residual pairwise networks for one shot learning, arXiv preprint arXiv:1703.08033.

[84] I. Goodfellow, J. Pouget-Abadie, M. Mirza, B. Xu, D. Warde-Farley, S. Ozair, A. Courville, Y. Bengio, Generative adversarial nets, in: Advances in neural information processing systems, 2014, pp. 2672-2680.

[85] T. Schlegl, P. Seeböck, S. M. Waldstein, U. Schmidt-Erfurth, G. Langs, Unsupervised anomaly detection with generative adversarial networks to guide marker discovery, in: International Conference on Information Processing in Medical Imaging, Springer, 2017, pp. 146-157.

[86] J. Canny, A computational approach to edge detection, IEEE Transactions on Pattern Analysis and Machine Intelligence PAMI-8 (6) (1986) 679698. doi:10.1109/TPAMI.1986.4767851.

[87] G. Turin, An introduction to matched filters, IRE transactions on Information theory 6 (3) (1960) 311-329.

[88] J. Radon, On the determination of functions from their integral values along certain manifolds, IEEE transactions on medical imaging 5 (4) (1986) 170-176.

[89] S. Lloyd, Least squares quantization in pcm, IEEE transactions on information theory 28 (2) (1982) 129-137.

[90] J. C. Dunn, A fuzzy relative of the isodata process and its use in detecting compact well-separated clusters, Cybernetics 3 (1973) 32-57.

[91] J. Wagner, V. Fischer, M. Herman, S. Behnke, Multispectral pedestrian detection using deep fusion convolutional neural networks, in: 24th European Symposium on Artificial Neural Networks, Computational Intelligence and Machine Learning (ESANN), 2016, pp. 509-514.

[92] R. Gade, T. B. Moeslund, Thermal cameras and applications: A survey, Machine vision and applications 25 (1) (2014) 245-262.

[93] S. Sorensen, W. Treible, L. Hsu, X. Wang, A. R. Mahoney, D. P. Zitterbart, C. Kambhamettu, Deep learning for polar bear detection, in: Scandinavian Conference on Image Analysis, Springer, 2017, pp. 457-467.

[94] J. Liu, S. Zhang, S. Wang, D. N. Metaxas, Multispectral deep neural networks for pedestrian detection, arXiv preprint arXiv:1611.02644.

[95] D. König, M. Adam, C. Jarvers, G. Layher, H. Neumann, M. Teutsch, Fully convolutional region proposal networks for multispectral person detection, in: IEEE Conference on Computer Vision and Pattern Recognition Workshops (CVPRW), IEEE, 2017, pp. 243-250. 